\title{
Practical and Reliable Error Bars in Quantum Tomography
}

\author{
Philippe Faist ${ }^{*}$ and Renato Renner \\ Institute for Theoretical Physics, ETH Zurich, 8093 Zurich, Switzerland
}

(Received 18 March 2016; published 1 July 2016)

\begin{abstract}
Precise characterization of quantum devices is usually achieved with quantum tomography. However, most methods which are currently widely used in experiments, such as maximum likelihood estimation, lack a welljustified error analysis. Promising recent methods based on confidence regions are difficult to apply in practice or yield error bars which are unnecessarily large. Here, we propose a practical yet robust method for obtaining error bars. We do so by introducing a novel representation of the output of the tomography procedure, the quantum error bars. This representation is (i) concise, being given in terms of few parameters, (ii) intuitive, providing a fair idea of the "spread" of the error, and (iii) useful, containing the necessary information for constructing confidence regions. The statements resulting from our method are formulated in terms of a figure of merit, such as the fidelity to a reference state. We present an algorithm for computing this representation and provide ready-to-use software. Our procedure is applied to actual experimental data obtained from two superconducting qubits in an entangled state, demonstrating the applicability of our method.
\end{abstract}

DOI: 10.1103/PhysRevLett.117.010404

Introduction.-Recent experimental developments have demonstrated increasingly precise manipulation and control of quantum systems, paving the way towards the hopeful implementation of a quantum computer [1-12]. The successful outcome of an experiment is usually certified using quantum tomography. This is the task of inferring the quantum state of a device from statistics of measurements on many copies of the system [13-19]. Several methods perform this task and are widely used, such as maximum likelihood estimation [20,21].

In the realistic regime where finite data are collected, the error bars provided by most methods which are widely applied in current experiments [19,22-24] are typically ill justified and may lead to deceiving conclusions [25-27]. To remedy this problem, Blume-Kohout [27] and Christandl and Renner [28] resort to confidence regions. These are regions in state space of all density matrices in which the state lies with high probability. In contrast to Bayesian methods [25], the reliability statements do not depend on any prior distributions. However, confidence regions are a priori difficult to construct explicitly [29]. Furthermore, they are designed for worst-case scenarios and are often not representative of the intuitive extent of the error.

Our main result is a novel representation of the output of the tomography procedure - a summary of what the tomographic data tells us about the state of the system-which we call quantum error bars. This description is (i) concise, being given in terms of a few parameters only, (ii) intuitive,

Published by the American Physical Society under the terms of the Creative Commons Attribution 3.0 License. Further distribution of this work must maintain attribution to the author(s) and the published article's title, journal citation, and DOI. providing a fair idea of the "spread" of the error, and (iii) useful for precise statements, containing all necessary information for constructing confidence regions. Our method particularly inherits the mathematical robustness of the confidence region approach.

The quantum error bars are designed to mimic the role of classical error bars. Classically, an error bar typically represents the standard deviation of the distribution of a physical quantity caused by noise or statistical errors; this distribution is usually assumed to be Gaussian. Observe that, precisely, classical error bars (i) are a concise description of the error, (ii) provide a fair, intuitive idea of the spread of the quantity of interest, and (iii) allow us to calculate precise statements such as the required error interval to consider (e.g., 5 standard deviations) for a specific requested certainty level (e.g., one in a million).

Our statements are formulated in terms of a figure of merit which can be chosen freely. Our method works best when the figure of merit is the fidelity to a pure target state, the expectation value of an observable, or the trace distance to any reference state. This encompasses most tomography settings.

The quantum error bars are constructed as follows. The input is the experimental data from a general quantum tomography experiment. Then we construct a particular distribution $\mu(f)$ of the chosen figure of merit $f$, which has the property of containing the necessary information to construct confidence regions at any confidence level using the method of Ref. [28]. We show that in a wide range of situations and for a class of figures of merit, the distribution $\mu(f)$ can be approximated by a simple analytical expression with three parameters. The quantum error bars are then straightforwardly deduced from these parameters.

We provide a simple numerical algorithm to obtain the quantum error bars from the measurement data. By fitting a 


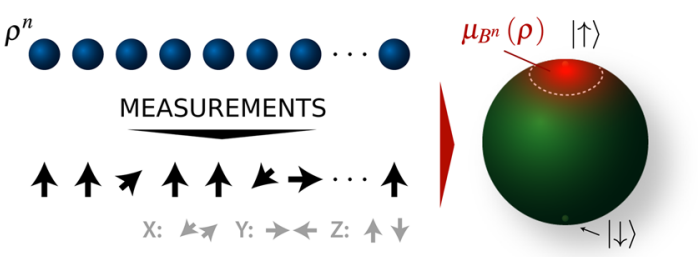

FIG. 1. Setup of quantum tomography. Measurements are taken on $n$ copies of a quantum system. The outcomes allow us to infer what the state of the quantum system is. In this example a qubit is measured using Pauli operators. Here, the experimental data are most consistent with the state being $|\uparrow\rangle$, located at the top of the Bloch sphere (in green). However, because only finite data are collected, there is an uncertainty associated with this statement. In the method of Ref. [28], a distribution $\mu_{B^{n}}(\rho)$ (the red gradient) is determined from the data, from which confidence regions can be constructed (delimited by the dotted line). These are regions in state space in which the state lies with high probability.

+numerical approximation of $\mu(f)$ with our approximate analytical model, we obtain the values of the parameters of the model which directly translate to the quantum error bars. The practicality of our method is demonstrated by applying it to experimental data from two superconducting qubits.

Our work complements a vast literature which has provided error analyses for experiments [30-42], as well as explicit schemes [43-52], by introducing the novel concept of quantum error bars. The complexity of such schemes have also been investigated $[53,54]$ and numerical techniques put forward [25,55-57]. Furthermore, a number of contributions propose measurement schemes for fidelity estimation $[58,59]$, tomography of matrix product states [60], estimation of low-rank states [61,62], and permutationally invariant tomography [63-65]. An experiment following such schemes would achieve target benchmarks more efficiently, and it could still be analyzed using our procedure, the latter being applicable to any measurements.

The rest of this Letter is structured as follows. First, we briefly explain our quantum tomography setup and the concept of confidence regions. We then derive our main technical results, namely, the definition of $\mu(f)$, its approximate theoretical model, and the algorithm to estimate $\mu(f)$ numerically. Finally, we demonstrate the applicability of our method on experimental data.

Quantum tomography setup.-A large number $n$ of copies of a quantum system are measured using independent, possibly different, measurement settings (Fig. 1) [66]. We list all of the distinct positive operator valued measure (POVM) effects in one set $\left\{E_{k}\right\}$, and denote by $n_{k}$ the number of times the POVM effect $E_{k}$ was observed. We then construct the likelihood function, which will be needed in our analysis. It is defined as the probability with which the observed data would occur if the true state were $n$ copies of $\rho$,

$$
\Lambda(\rho)=\operatorname{Pr}[\text { observed data } \mid \rho]=\prod_{k}\left(\operatorname{tr}\left[E_{k} \rho\right]\right)^{n_{k}},
$$

along with the log-likelihood,

$$
\lambda(\rho)=-2 \ln \Lambda(\rho)=-2 \sum_{k} n_{k} \ln \operatorname{tr}\left(E_{k} \rho\right),
$$

with a conventional $(-2)$ factor $[27,33]$.

Confidence regions.-In the following, we briefly review the method of Ref. [28] for constructing confidence regions, on which our method is based.

Confidence regions of confidence level $1-\epsilon$ are defined as regions in state space which contain the true state with a probability of at least $1-\epsilon$. Crucially, it is the complete procedure of assigning a region to tomographic data which is certified and not the particular region itself (despite the slightly misleading terminology). More precisely, for a particular "true" state $\rho_{\text {true }}$, the measurement outcomes observed in the tomography procedure are only one possible outcome data set among the enormous amount of theoretically possible data sets. Now, a data analysis procedure associates with each observed data set a corresponding region in state space. This tomography procedure is said to yield confidence regions of confidence level $1-\epsilon$ if, for any true state $\rho_{\text {true }}$, the tomography procedure associates with the observed data set a region which contains $\rho_{\text {true }}$, except for some data sets with the total probability $\epsilon$. In other words, the complete tomography procedure is successful except with the probability $\epsilon$, in which case the observed data set may cause the procedure to report a bad region. These "exceptional data sets" may be interpreted as misleading but highly unlikely situations. For example, if we flip a fair coin many times and observe the sequence of all "heads," any reasonable inference scheme would wrongly report that the coin is highly biased. However this outcome only happens with disproportionately small probability; introducing the parameter $\epsilon$ above allows us to disregard such extremely unlikely cases.

The method of Ref. [28] is formulated using the estimate density $\mu_{B^{n}}$ [82], defined as

$$
\mu_{B^{n}}(\rho)=\frac{1}{c_{B^{n}}} \Lambda(\rho)
$$

where $c_{B^{n}}$ is a normalizing factor such that $\int d \rho \mu_{B^{n}}(\rho)=1$, and where $d \rho$ is the Hilbert-Schmidt measure normalized such that $\int d \rho=1[83,84]$. The main result of Ref. [28] is a criterion for certifying a procedure for yielding confidence regions of confidence level $1-\epsilon$. The criterion is the following: the procedure should map to any tomographic data (essentially) a region $R$ in state space which satisfies

$$
\int_{R} \mu_{B^{n}}(\rho) d \rho=1-\frac{\epsilon}{\operatorname{poly}(n)},
$$

i.e., which has high weight under the distribution $\mu_{B^{n}}$ [85]. 
Confidence regions for a figure of merit.-We may now use this criterion to devise an explicit procedure for constructing confidence regions, where the regions $R$ are chosen to be defined via level sets of a figure of merit.

A figure of merit $f(\rho)$ may be any function of the quantum state. For example, $f(\rho)=F^{2}\left(\rho,\left|\psi_{\text {Ref }}\right\rangle\left\langle\psi_{\text {Ref }}\right|\right)$ expresses the fidelity to a reference state $\left|\psi_{\text {Ref }}\right\rangle$. The reduced distribution of the estimate density $\mu_{B^{n}}(\rho)$ onto the figure of merit $f$ is given by

$$
\mu(f)=\int d \rho \mu_{B^{n}}(\rho) \delta(f(\rho)-f),
$$

where $\delta(\cdot)$ denotes the Dirac delta function.

Now fix a threshold value $f$, and consider the region $R_{f}$ in state space consisting of all states whose figure of merit is greater than or equal to $f$ (Fig. 2). The weight of the region $R_{f}$ according to the distribution $\mu_{B^{n}}(\rho)$ is exactly given by $\int_{f^{\prime} \geq f} \mu\left(f^{\prime}\right) d f^{\prime}$. Inverting this reasoning, for any $\epsilon$, we can find the maximum threshold value $f$ required for a region $R_{f}$ to encompass a particular weight $1-\epsilon / \operatorname{poly}(n)$; we know that this region is essentially a confidence region by the criterion of Ref. [28]. [If the figure of merit is such that smaller values of $f(\rho)$ are desirable, such as the trace distance to a reference state, then $R_{f}$ is defined with $f$ as an upper, rather than lower, threshold value].

We arrive at a first important observation: if we find a simple characterization of the function $\mu(f)$, then we are capable of constructing confidence regions in terms of $f$ for any confidence level [86].

Determining $\mu(f)$ numerically.-We propose a practical procedure which determines a numerical estimate of $\mu(f)$. We resort to a Monte Carlo-type scheme known as the Metropolis-Hastings algorithm [87] (cf. also Refs. [88,89]). This algorithm is a standard, well-tested scheme widely used in computational physics-for instance, to simulate the behavior of statistical systems at finite temperature [90] - and there are standard methods for controlling the uncertainties resulting from this procedure [91]. Using this algorithm, we conduct a random walk in the quantum state space and produce random samples distributed according to the distribution $\mu_{B^{n}}(\rho)$. By collecting the values of $f(\rho)$ at

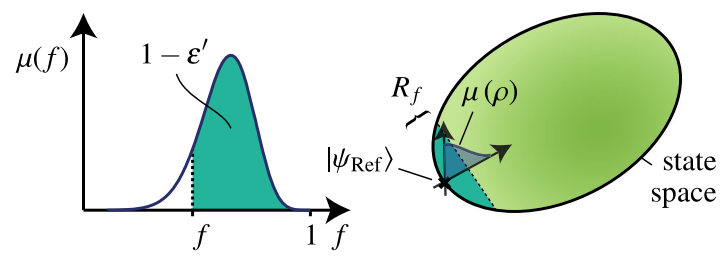

FIG. 2. Construction of confidence regions from the distribution $\mu(f)$ on the figure of merit. High weight intervals with respect to $\mu(f)$ (left plot) correspond to high weight regions in state space with respect to $\mu_{B^{n}}(\rho)$ (right diagram) which are (essentially) confidence regions, according to Ref. [28]. the sampled points into a histogram, we obtain an estimate for $\mu(f)$. (See the Supplemental Material [66] for details on the random walk procedure).

Theoretical model for $\mu(f)$. - It turns out that, for a selection of common figures of merit, we may understand the numerical estimate of $\mu(f)$ with a theoretical model. Suppose $f(\rho)$ is the fidelity to a pure reference state, the expectation value of an observable, or the trace distance to any reference state. Then, under some reasonable assumptions [92], we derive the following approximate theoretical model for $\mu(f)$ (see the Supplemental Material [66]):

$$
\mu(f) \approx C(f-h)^{m} e^{-a_{2}(f-h)^{2}-a_{1}(f-h)},
$$

with three fit parameters, $a_{1}, a_{2}$, and $m$ (with $m \geq 0$ ), and one constant normalization factor $C$; $h$ is a constant depending only on the choice of the figure of merit. Specific values of the constant $h$ for some figures of merit are summarized in Table I.

The parameters $\left(a_{2}, a_{1}, m\right)$ are then mapped onto new parameters which are more representative of the shape of the function. The latter is viewed as a "skewed" Gaussian (see the Supplemental Material [66]). The parameter $f_{0}$ determines the position of the peak, the parameter $\Delta$ is the half width of the "deskewed" Gaussian, and $\gamma$ characterizes the deviation from a perfect Gaussian. The parameters $\left(f_{0}, \Delta, \gamma\right)$ are the quantum error bars.

Application to experimental data.-We have applied the algorithm to experimental data from two superconducting qubits prepared in a Bell state according to the setup described in Refs. [10,93]. The data were kindly provided by the authors of Ref. [10]. The two qubits were measured using slightly noisy individual Pauli operators, with a total of $n=55677$ measurements. The numerical estimation of $\mu(f)$ corresponding to the fidelity to the target Bell state is depicted in Fig. 3. (See the Supplemental Material [66] for

TABLE I. Theoretical fit model for some selected figures of merit. Here, $\left|\psi_{\text {Ref }}\right\rangle$ denotes any pure state, and $\rho_{\text {Ref }}$ any pure or mixed state. We use the notation $D(\rho, \sigma)$ for the trace distance and $\langle A\rangle_{\rho}=\operatorname{tr}(A \rho)$ for the expectation value of an observable $A$. The value $a$ is an extremal value of $\langle A\rangle_{\rho}$ for valid density matrices $\rho$ close to the region of interest, and $x$ should be chosen as $x=a-f(x=f-a)$ if $a$ is a maximal value (minimal value). If the extremum point of $A$ is far from the region of interest, the logarithm term in the model can be dropped, as the exponential will dominate the volume term, and $a$ can be absorbed into the other factors.

$$
\ln \mu(f) \approx-a_{2} x^{2}-a_{1} x+m \ln x+c, \text { where }
$$

\begin{tabular}{lc} 
Figure of merit $f(\rho)$ & $x=$ \\
\hline$F^{2}\left(\rho,\left|\psi_{\text {Ref }}\right\rangle\left\langle\psi_{\text {Ref }}\right|\right)=\left\langle\psi_{\text {Ref }}|\rho| \psi_{\text {Ref }}\right\rangle$ & $1-f$ \\
$D\left(\rho, \rho_{\text {Ref }}\right)=\frac{1}{2}\left\|\rho-\rho_{\text {Ref }}\right\|_{1}$ & $f$ \\
Observable $\langle A\rangle_{\rho}$ & $a-f$ or $f-a$ \\
\hline \hline
\end{tabular}




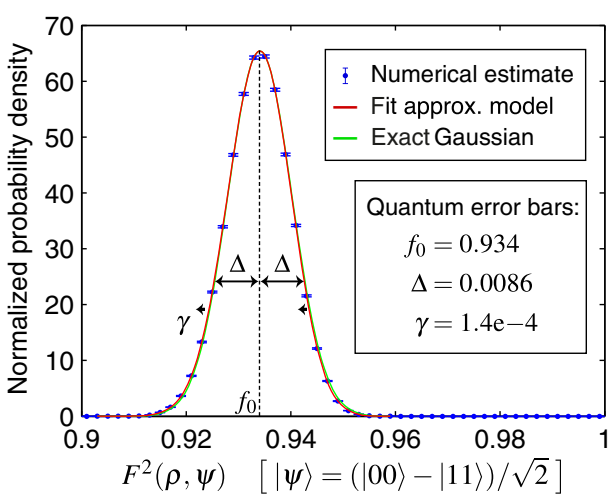

FIG. 3. Analysis of measurement data from two superconducting qubits prepared in a Bell state. We determined effective measurement operators which model the noise in the measurement process. The histogram of the fidelity to the target state $|\psi\rangle$ (the blue data points), produced using our procedure, fits well to our theoretical model in Table I. The quantum error bars are a concise, intuitive, and precise characterization of the fit model, which is interpreted as a skewed Gaussian function. The parameter $f_{0}$ is the peak maximum, $\Delta$ is the half width of the original Gaussian, and $\gamma$ characterizes the skewing in terms of the displacement of the sides of the peak from the exact Gaussian at the relative height $1 / e$. This example involving experimental data demonstrates a good level of practical applicability of our method.

details of the analysis of the experiment, including the modeling of the measurements [94] into effective POVM operators).

Quantum error bars.-The quantum error bars $\left(f_{0}, \Delta, \gamma\right)$ displayed in Fig. 3, are a concise and useful description of the error analysis, from which reliable operational statements can be made. Indeed, they provide the necessary information for constructing confidence regions for any given confidence level.

Also, as seen in Fig. 3, our error bars have the intuitive interpretation as representing the spread of the figure of merit according to $\mu(f)$. As such, the error bars are much smaller than the size of a confidence region for a small epsilon in the worst-case scenario, and they are in fact of comparable size to those obtained by bootstrapping [22,24,27,41,95] (see the Supplemental Material [66]).

Discussion.-Our work bridges the apparent gap between carrying out a mathematically rigorous, welljustified error analysis and using an ad hoc procedure yielding smaller error bars. The quantum error bars provide a convenient and precise representation of the information provided by the tomography procedure.

While the fit model for $\mu(f)$ is subject to some assumptions and approximations, it applies well to many examples studied by the authors in developing this workfor $n \sim 100$ total measurements already-and has been tested with up to five qubits. Note that the numerical procedure is not subject to these assumptions, and a deviation from the fit model could easily be noticed in some extreme examples considered (for example, with goodness-of-fit measures). A further detailed discussion on the reliability of our method is presented in the Supplemental Material [66].

It is relatively straightforward to apply our method to experimental setups consisting of a few qubits. Our procedure is restricted neither to particular measurement settings nor to specific quantum states, and it applies, for example, to adaptive tomography. In general, noise in the measurement procedure has to be modeled into effective POVM effects analogously to our approach for the two superconducting qubits. (In contrast, other approaches do not require this [96-98]). We have developed a software which implements our procedure [99] that is expected to be directly applicable to most experimental settings.

For worst-case scenarios such as quantum cryptography [100], it is still desirable to improve the methods for explicitly constructing confidence regions. We do anticipate that the bounds used in Ref. [28] may be tightened to yield smaller confidence regions for the same confidence level. If the construction is not altered, the procedure presented here would not require any change, as the same histograms may still serve for constructing confidence regions using the tightened proof.

We also insist that our results do not rely on any particular interpretation of "probability," such as a Bayesian or frequentist one. This is because we consider experiments which can, in principle, be repeated arbitrarily many times, which is a regime where these interpretations are equivalent [28]. Nonetheless, the Bayesian viewpoint is convenient, as the distribution $\mu(f)$ happens to coincide with the Bayesian posterior corresponding to an agent starting the tomography procedure with a Hilbert-Schmidt uniform prior.

Furthermore, even though our results are formulated in the context of quantum state tomography, the same procedure may be applied to quantum process tomography $[101,102]$. Indeed, the Choi-Jamiołkowski isomorphism implies that determining a quantum process is mathematically the same as determining a bipartite quantum state.

We thank Robin Blume-Kohout, Matthias Christandl, Steve Flammia, Aleksejs Fomins, Olivier Landon-Cardinal, Romain Müller, Denis Rosset, Cyril Stark, Lars Steffen, and Takanori Sugiyama for the fruitful discussions. We acknowledge support from the European Research Council (ERC) via Grant No. 258932, from the Swiss National Science Foundation through the National Centre of Competence in Research "Quantum Science and Technology" (QSIT), and by the European Commission via the project "RAQUEL".

*pfaist@phys.ethz.ch

[1] C. H. Bennett and D. P. DiVincenzo, Nature (London) 404, 247 (2000).

[2] J. P. Home, in Advances In Atomic, Molecular, and Optical Physics, edited by E. Arimondo, P. R. Berman, and C. C. Lin (Elsevier, New York, 2013), Vol. 62, p. 231. 
[3] M. H. Devoret and R. J. Schoelkopf, Science 339, 1169 (2013).

[4] T. E. Northup and R. Blatt, Nat. Photonics 8, 356 (2014).

[5] M. F. Riedel, P. Böhi, Y. Li, T. W. Hänsch, A. Sinatra, and P. Treutlein, Nature (London) 464, 1170 (2010).

[6] T. Monz, P. Schindler, J. T. Barreiro, M. Chwalla, D. Nigg, W. A. Coish, M. Harlander, W. Hänsel, M. Hennrich, and R. Blatt, Phys. Rev. Lett. 106, 130506 (2011).

[7] P. C. Maurer, G. Kucsko, C. Latta, L. Jiang, N. Y. Yao, S. D. Bennett, F. Pastawski, D. Hunger, N. Chisholm, M. Markham, D. J. Twitchen, J. I. Cirac, and M. D. Lukin, Science 336, 1283 (2012).

[8] I. Usmani, C. Clausen, F. Bussières, N. Sangouard, M. Afzelius, and N. Gisin, Nat. Photonics 6, 234 (2012).

[9] A. Fedorov, L. Steffen, M. Baur, M. P. da Silva, and A. Wallraff, Nature (London) 481, 170 (2012).

[10] L. Steffen, Y. Salathe, M. Oppliger, P. Kurpiers, M. Baur, C. Lang, C. Eichler, G. Puebla-Hellmann, A. Fedorov, and A. Wallraff, Nature (London) 500, 319 (2013).

[11] F. Bussières, C. Clausen, A. Tiranov, B. Korzh, V. B. Verma, S. W. Nam, F. Marsili, A. Ferrier, P. Goldner, H. Herrmann, C. Silberhorn, W. Sohler, M. Afzelius, and N. Gisin, Nat. Photonics 8, 775 (2014).

[12] T. P. Harty, D. T. C. Allcock, C. J. Ballance, L. Guidoni, H. A. Janacek, N. M. Linke, D. N. Stacey, and D. M. Lucas, Phys. Rev. Lett. 113, 220501 (2014).

[13] C. W. Helstrom, J. Stat. Phys. 1, 231 (1969).

[14] K. Vogel and H. Risken, Phys. Rev. A 40, 2847 (1989).

[15] G. M. D'Ariano, Phys. Lett. A 268, 151 (2000).

[16] G. Cassinelli, G. M. D'Ariano, E. De Vito, and A. Levrero, J. Math. Phys. (N.Y.) 41, 7940 (2000).

[17] R. T. Thew, A. G. White, and W. J. Munro, Phys. Rev. A 66, 012303 (2002).

[18] G. M. D’Ariano, M. G. A. Paris, and M. F. Sacchi, Adv. Imaging Electron Phys. 128, 205 (2003).

[19] Quantum State Estimation, Lecture Notes in Physics Vol. 649, edited by M. Paris and J. Řeháček (Springer, Berlin, 2004).

[20] Z. Hradil, Phys. Rev. A 55, R1561 (1997).

[21] J. Řeháček, Z. Hradil, and M. Ježek, Phys. Rev. A 63, 040303 (2001).

[22] B. Efron and R. J. Tibshirani, An Introduction to the Bootstrap (CRC Press, Boca Raton, FL, 1994).

[23] D. F. V. James, P. G. Kwiat, W. J. Munro, and A. G. White, Phys. Rev. A 64, 052312 (2001).

[24] J. P. Home, D. Hanneke, J. D. Jost, J. M. Amini, D. Leibfried, and D. J. Wineland, Science 325, 1227 (2009).

[25] R. Blume-Kohout, New J. Phys. 12, 043034 (2010).

[26] B. Jungnitsch, S. Niekamp, M. Kleinmann, O. Gühne, H. Lu, W.-B. Gao, Y.-A. Chen, Z.-B. Chen, and J.-W. Pan, Phys. Rev. Lett. 104, 210401 (2010).

[27] R. Blume-Kohout, Report No. LA-UR-12-00824.

[28] M. Christandl and R. Renner, Phys. Rev. Lett. 109, 120403 (2012).

[29] J. M. Arrazola, O. Gittsovich, J. M. Donohue, J. Lavoie, K. J. Resch, and N. Lütkenhaus, Phys. Rev. A 87, 062331 (2013).

[30] T. Kiesel, W. Vogel, V. Parigi, A. Zavatta, and M. Bellini, Phys. Rev. A 78, 021804 (2008).
[31] J. Řeháček, D. Mogilevtsev, and Z. Hradil, New J. Phys. 10, 043022 (2008).

[32] T. Kiesel and W. Vogel, Phys. Rev. A 82, 032107 (2010).

[33] R. Blume-Kohout, J. O. S. Yin, and S. J. van Enk, Phys. Rev. Lett. 105, 170501 (2010).

[34] R. Blume-Kohout, Phys. Rev. Lett. 105, 200504 (2010).

[35] T. Sugiyama, P. Turner, and M. Murao, Phys. Rev. A 83, 012105 (2011).

[36] C. Ferrie and R. Blume-Kohout, AIP Conf. Proc. 1443, 14 (2012).

[37] D. Rosset, R. Ferretti-Schöbitz, J.-D. Bancal, N. Gisin, and Y.-C. Liang, Phys. Rev. A 86, 062325 (2012).

[38] J. Shang, H. K. Ng, A. Sehrawat, X. Li, and B.-G. Englert, New J. Phys. 15, 123026 (2013).

[39] M. Walter and J. M. Renes, IEEE Trans. Inf. Theory 60, 8007 (2014).

[40] T. Sugiyama, Phys. Rev. A 91, 042126 (2015).

[41] C. Schwemmer, L. Knips, D. Richart, H. Weinfurter, T. Moroder, M. Kleinmann, and O. Gühne, Phys. Rev. Lett. 114, 080403 (2015).

[42] H. Ball, T. M. Stace, S. T. Flammia, and M. J. Biercuk, Phys. Rev. A 93, 022303 (2016).

[43] A. I. Lvovsky, J. Opt. B 6, S556 (2004).

[44] R. Blume-Kohout and P. Hayden, arXiv:quant-ph/0603116.

[45] R. Schmied and P. Treutlein, New J. Phys. 13, 065019 (2011).

[46] K. Dobek, M. Karpiński, R. Demkowicz-Dobrzański, K. Banaszek, and P. Horodecki, Phys. Rev. Lett. 106, 030501 (2011).

[47] J. Smolin, J. Gambetta, and G. Smith, Phys. Rev. Lett. 108, 070502 (2012).

[48] A. Anis and A. I. Lvovsky, New J. Phys. 14, 105021 (2012).

[49] R. D. Gill and M. I. Gută, in From Probability to Statistics and Back: High-Dimensional Models and Processes-A Festschrift in Honor of Jon A. Wellner, Institute of Mathematical Statistics Collections Vol. 9, edited by M. Banerjee, F. Bunea, J. Huang, V. Koltchinskii, and M. H. Maathuis (Institute of Mathematical Statistics, Beachwood, OH, 2013), p. 105.

[50] M. Kech, P. Vrana, and M. M. Wolf, J. Phys. A 48, 265303 (2015).

[51] G. Haack, A. Steffens, J. Eisert, and R. Hübener, New J. Phys. 17, 113024 (2015).

[52] A. Carpentier, J. Eisert, D. Gross, and R. Nickl, arXiv: 1504.03234.

[53] J. Haah, A. W. Harrow, Z. Ji, X. Wu, and N. Yu, Proceedings of the 48th Annual ACM SIGACT Symposium on Theory of Computing, 2016 (ACM, New York, NY, USA, 2016), pp. 913-925.

[54] R. O'Donnell and J. Wright, Proceedings of the 48th Annual ACM SIGACT Symposium on Theory of Computing (ACM, New York, NY, USA, 2016), pp. 899-912.

[55] J. Řeháček, Z. Hradil, E. Knill, and A. Lvovsky, Phys. Rev. A 75, 042108 (2007).

[56] R. Kueng and C. Ferrie, New J. Phys. 17, 123013 (2015).

[57] C. Granade, J. Combes, and D. G. Cory, New J. Phys. 18, 033024 (2016).

[58] M. P. da Silva, O. Landon-Cardinal, and D. Poulin, Phys. Rev. Lett. 107, 210404 (2011). 
[59] S. T. Flammia and Y.-K. Liu, Phys. Rev. Lett. 106, 230501 (2011).

[60] M. Cramer, M. B. Plenio, S. T. Flammia, R. Somma, D. Gross, S. D. Bartlett, O. Landon-Cardinal, D. Poulin, and Y.-K. Liu, Nat. Commun. 1, 149 (2010).

[61] D. Gross, Y.-K. Liu, S. Flammia, S. Becker, and J. Eisert, Phys. Rev. Lett. 105, 150401 (2010).

[62] S. T. Flammia, D. Gross, Y.-K. Liu, and J. Eisert, New J. Phys. 14, 095022 (2012).

[63] G. Tóth, W. Wieczorek, D. Gross, R. Krischek, C. Schwemmer, and H. Weinfurter, Phys. Rev. Lett. 105, 250403 (2010).

[64] T. Moroder, P. Hyllus, G. Tóth, C. Schwemmer, A. Niggebaum, S. Gaile, O. Gühne, and H. Weinfurter, New J. Phys. 14, 105001 (2012).

[65] C. Schwemmer, G. Tóth, A. Niggebaum, T. Moroder, D. Gross, O. Gühne, and H. Weinfurter, Phys. Rev. Lett. 113, 040503 (2014).

[66] See Supplemental Material at http://link.aps.org/ supplemental/10.1103/PhysRevLett.117.010404, which includes Refs. [67-81], for a discussion of the most general case in which the measurements need not be independent, and where the underlying quantum state of the $n$ systems only needs to be permutation invariant.

[67] R. A. Bertlmann and P. Krammer, J. Phys. A 41, 235303 (2008).

[68] E. Brüning, H. Mäkelä, A. Messina, and F. Petruccione, J. Mod. Opt. 59, 1 (2012).

[69] G. Kimura, Phys. Lett. A 314, 339 (2003).

[70] M.S. Byrd and N. Khaneja, Phys. Rev. A 68, 062322 (2003).

[71] M. Tomamichel, R. Colbeck, and R. Renner, IEEE Trans. Inf. Theory 56, 4674 (2010).

[72] M. M. Wilde, Quantum Information Theory (Cambridge University Press, Cambridge, England, 2013), p. 642.

[73] M. Christandl (private communication).

[74] A. Alexandrescu, Modern C++ Design: Generic Programming and Design Patterns Applied (AddisonWesley Professional, Reading, MA, 2001), p. 323.

[75] G. Guennebaud, B. Jacob et al., EIGEN, version 3, 2010 http://eigen.tuxfamily.org/.

[76] Boost libraries, http://www.boost.org (2015).

[77] M. Grant and S. Boyd, in Recent Advances in Learning and Control, Lecture Notes in Control and Information Sciences Vol. 371, edited by V. Blondel, S. Boyd, and H. Kimura (Springer-Verlag, Berlin, 2008), p. 95.

[78] M. Grant and S. Boyd, CVX: MATLAB software for disciplined convex programming, version 2.1, http://cvxr .com/cvx (2014).

[79] Y. Teo, B.-G. Englert, J. Řeháček, and Z. Hradil, Phys. Rev. A 84, 062125 (2011).

[80] T. Sugiyama, P. S. Turner, and M. Murao, Phys. Rev. A 85, 052107 (2012).
[81] D. H. Mahler, L. A. Rozema, A. Darabi, C. Ferrie, R. Blume-Kohout, and A. M. Steinberg, Phys. Rev. Lett. 111, 183601 (2013).

[82] We use the notation of Ref. [28]; there, the general case of a joint measurement on the $n$ systems is considered and the outcome POVM effect is denoted by $B^{n}$.

[83] I. Bengtsson and K. Zyczkowski, Geometry of Quantum States: An Introduction to Quantum Entanglement (Cambridge University Press, Cambridge, England, 2006).

[84] K. Zyczkowski and H.-J. Sommers, J. Phys. A 34, 7111 (2001).

[85] The output region should in fact be $R^{\delta}$, obtained by enlarging the set $R$ by some small $\delta$ in fidelity distance; explicit expressions for $\operatorname{poly}(n)$ and $\delta$ are given in Ref. [28].

[86] See Supplemental Material at http://link.aps.org/ supplemental/10.1103/PhysRevLett.117.010404 for ways to transpose the $\delta$ enlargement in Ref. [28] into a shift of the threshold value $f$.

[87] N. Metropolis, A. W. Rosenbluth, M. N. Rosenbluth, A. H. Teller, and E. Teller, J. Chem. Phys. 21, 1087 (1953).

[88] J. Shang, Y.-L. Seah, H. K. Ng, D. J. Nott, and B.-G. Englert, New J. Phys. 17, 043017 (2015).

[89] Y.-L. Seah, J. Shang, H. K. Ng, D. J. Nott, and B.-G. Englert, New J. Phys. 17, 043018 (2015).

[90] A. F. Albuquerque et al., J. Magn. Magn. Mater. 310, 1187 (2007).

[91] V. Ambegaokar and M. Troyer, Am. J. Phys. 78, 150 (2010).

[92] We assume that not too few measurements have been taken, and we invoke some approximation methods, such as Laplace's method, for integrating an exponential (see details in the Supplemental Material [66]).

[93] M. Baur, A. Fedorov, L. Steffen, S. Filipp, M. P. Da Silva, and A. Wallraff, Phys. Rev. Lett. 108, 040502 (2012).

[94] R. Bianchetti, S. Filipp, M. Baur, J. Fink, M. Göppl, P. Leek, L. Steffen, A. Blais, and A. Wallraff, Phys. Rev. A 80, 043840 (2009).

[95] M. W. Mitchell, C. W. Ellenor, S. Schneider, and A. M. Steinberg, Phys. Rev. Lett. 91, 120402 (2003).

[96] C. Stark, Phys. Rev. A 89, 052109 (2014).

[97] C. Stark, arXiv:1209.6499.

[98] C. Stark, arXiv:1210.1105.

[99] P. Faist, The tomographer project, https://github.com/ Tomographer/tomographer (2015).

[100] M. Tomamichel, C. C. W. Lim, N. Gisin, and R. Renner, Nat. Commun. 3, 634 (2012).

[101] I. L. Chuang and M. A. Nielsen, J. Mod. Opt. 44, 2455 (1997).

[102] J. L. O’Brien, G. J. Pryde, A. Gilchrist, D. F. V. James, N. K. Langford, T. C. Ralph, and A. G. White, Phys. Rev. Lett. 93, 080502 (2004). 